\title{
Exact Boundary Controllability for Free Traffic Flow with Lipschitz Continuous State
}

\author{
Martin Gugat \\ Department Mathematik, Friedrich-Alexander-Universität Erlangen-Nürnberg (FAU), Cauerstraße 11, 91058 Erlangen, Germany \\ Correspondence should be addressed to Martin Gugat; martin.gugat@fau.de
}

Received 3 December 2015; Accepted 21 March 2016

Academic Editor: Chaudry Masood Khalique

Copyright (C) 2016 Martin Gugat. This is an open access article distributed under the Creative Commons Attribution License, which permits unrestricted use, distribution, and reproduction in any medium, provided the original work is properly cited.

\begin{abstract}
We consider traffic flow governed by the LWR model. We show that a Lipschitz continuous initial density with free-flow and sufficiently small Lipschitz constant can be controlled exactly to an arbitrary constant free-flow density in finite time by a piecewise linear boundary control function that controls the density at the inflow boundary if the outflow boundary is absorbing. Moreover, this can be done in such a way that the generated state is Lipschitz continuous. Since the target states need not be close to the initial state, our result is a global exact controllability result. The Lipschitz constant of the generated state can be made arbitrarily small if the Lipschitz constant of the initial density is sufficiently small and the control time is sufficiently long. This is motivated by the idea that finite or even small Lipschitz constants are desirable in traffic flow since they might help to decrease the speed variation and lead to safer traffic.
\end{abstract}

\section{Introduction}

The Lighthill Whitham and Richards (LWR) model is a macroscopic model for traffic flow (see $[1,2])$. In the model, the traffic flow is described by the solution of an initial boundary value problem with a hyperbolic partial differential equation (pde). The pde can be solved using the method of characteristics. In fact, since the pde is a scalar conservation law, for this model the characteristic curves are straight lines. A derivation of this macroscopic model as a limit of a microscopic model is given in [3].

In the control of traffic flow, for example, for the flow through a tunnel, it is desirable to have free-flow traffic in the tunnel where the density remains below a certain critical density that corresponds to maximal throughput in the system. In this way, congested flow in the tunnel is avoided. This can be achieved by controlling the traffic inflow into the tunnel; see $[4,5]$.

It is well known that the solutions of the LWR model can develop shocks in finite time. However, in order to decrease variations in speed among the cars and along the road solutions without shocks are desirable. In this paper we construct Lipschitz continuous controls that generate Lipschitz continuous states. This is motivated by the idea that the traffic safety is increased if the Lipschitz constant is decreased, since this might help to decrease the speed variation among vehicles on the road and as vehicles drive along the road, which in turn reduces the expected accident rate; see [6, 7]. Moreover, we hope that as the Lipschitz constant decreases, also the fuel efficiency of the traffic flow increases. Thus, the proposed controls point out possible improvements for the control of free-flow traffic that may help to improve the safety and fuel efficiency in the free-flow regime. However, in the example of tunnel traffic flow control there is a trade-off between decreasing the Lipschitz constant of the traffic flow density flow within the tunnel and possible longer waiting time of the drivers before they are allowed to enter the tunnel. The reason is that in order to generate Lipschitz continuous states we impose an upper bound on the rate of change of the controlled inflow traffic density. We want to emphasize that the method presented in this paper cannot dissolve existing congested flow within the tunnel.

Exact boundary controllability has been studied intensively for classical solution of quasilinear hyperbolic partial 
differential equations, in particular by Li and his group; see [8]. In this paper we want to consider solutions that are less regular, namely, solutions generated by Lipschitz continuous initial and boundary data. It turns out that the solutions can still be represented using the method of characteristics. Also in $[9,10]$ the exact controllability has been studied in the framework of classical solutions. Both studies focus on systems where the eigenvalues can change their sign. In [9] the St.-Venant system that is a model for the flow of water through channels is considered, whereas in [10] a general class of systems with vanishing characteristic speed is studied. In the LWR model the characteristic speed vanishes exactly at the critical density where the traffic flow changes from free flow to congested flow. Since our exact controllability is not restricted to desired states that are close to the initial state, we can consider it as a global exact controllability result within the free-flow regime. A result of this kind has also been presented in [11] for the St.-Venant system. In fact, the basic idea of the proof in [11] is similar to the proof presented here. However, in [11] classical solutions are considered.

In this paper we show how Lipschitz continuous control functions can be chosen in such a way that they generate Lipschitz continuous states and control the given initial density in finite time to any desired density below the critical density. While the density can be increased arbitrarily fast without generating shock, in order to decrease the density to a substantially smaller density some intermediate steps can be necessary. As an example, a piecewise linear controller is presented explicitly in Section 5 of the paper.

The paper has the following structure. In Section 2, the boundary control system that we want to consider is defined and a well-posedness result in the framework of solutions that are defined in the sense of characteristics is given. Based upon this result, in Section 3, we study the exact controllability of the system. We present a result on global exact boundary controllability to constant states with Lipschitz continuous solutions. To prove this result, we first show two results on local exact controllability. In Section 4 we present a sufficient condition for Lipschitz continuity of the generated states and provide Lipschitz constants explicitly. In Section 5, we summarize the constructed piecewise linear control functions in explicit form and describe the Lipschitz continuous states that they generate.

\section{The System}

First we introduce some notation for the flux function.

2.1. The Flux Function. Let a maximal density $\rho_{\max }>0$ be given. Let a nonzero concave function $f$ that maps $\left[0, \rho_{\max }\right]$ to $[0, \infty)$ with $f(0)=0$ and $f\left(\rho_{\max }\right)=0$ be given.

The function $f$ defines the fundamental diagram (see [12]) and serves as the flux function in our traffic flow model. Define the critical density $\rho_{c}$ as the smallest point where the function $f$ attains its maximum value on $\left[0, \rho_{\max }\right]$. Assume that $f$ is continuously differentiable on $\left[0, \rho_{c}\right]$. The concavity of $f$ implies that it is strictly increasing on $\left[0, \rho_{c}\right]$. Moreover, $f^{\prime}$ is decreasing on $\left[0, \rho_{c}\right]$.
Assume that $f^{\prime}$ is Lipschitz continuous on $\left[0, \rho_{c}\right]$ with the Lipschitz constant $L_{f^{\prime}}>0$; that is, for all $\rho_{1}, \rho_{2} \in\left[0, \rho_{c}\right]$ we have the Lipschitz inequality:

$$
\left|f^{\prime}\left(\rho_{1}\right)-f^{\prime}\left(\rho_{2}\right)\right| \leq L_{f^{\prime}}\left|\rho_{1}-\rho_{2}\right| .
$$

For $\varepsilon \in\left(0, \rho_{c}\right)$ define

$$
\underline{\Lambda}(\varepsilon)=\min _{\rho \in\left[0, \rho_{c}-\varepsilon\right]} f^{\prime}(\rho)=f^{\prime}\left(\rho_{c}-\varepsilon\right)>0 .
$$

Remark 1. Since in our analysis we only work in the uncongested phase, in fact only the assumptions on $\rho$ as a function defined on the interval $\left[0, \rho_{c}\right]$ are relevant for our analysis.

2.2. The Initial Boundary Value Problem. Let $l>0$ denote the length of the considered road section. In particular, this could be the length of a tunnel. Let a Lipschitz continuous initial density $\rho_{0} \in C([0, l])$ with values in $\left[0, \rho_{c}\right]$ and the Lipschitz constant $L_{\rho_{0}}$ be given. Let a time $T>0$ be given. Let a Lipschitz continuous boundary density $u \in C([0, T])$ with the Lipschitz constant $L_{u}$ be given. Consider the system:

$$
\text { (S) } \begin{cases}\rho(0, x)=\rho_{0}(x), & x \in(0, l) \\ \rho(t, 0)=u(t), & t \in(0, T) \\ \rho_{t}+(f(\rho))_{x}=0, & \text { on }[0, T] \times[0, l] .\end{cases}
$$

Theorem 3 contains sufficient conditions for the existence of a solution of (S) in the sense of the characteristic curves on the time interval $[0, T]$. To avoid the generation of shocks at $t=0$, it is assumed that $u(0)<\rho_{c}$ and that $u$ and $\rho_{0}$ are compatible in the sense that $u(0)=\rho_{0}(0)$. The sufficient conditions are stated in terms of the size of the Lipschitz constants for the initial density and the boundary density. Note that in Theorem 3 we do not control the growth of the Lipschitz constants during the evolution of the solution. The reason is that we only assume that the time $T$ is strictly smaller than the breaking time, which can be estimated quite accurately. The corresponding result for the Burgers equation has been stated in [13]. The size of the Lipschitz constants of the generated states is studied in Section 4. In Theorem 12 explicit representations for these Lipschitz constants are presented that are increasing functions of the Lipschitz constants of the initial density and the boundary density and also of the time $T$. To make sure that the solution exists on a given possibly large time interval $[0, T]$, the Lipschitz constants $L_{\rho_{0}}$ and $L_{u}$ must be sufficiently small. Note that the values of the boundary density and hence also the initial density at $x=0$ are assumed to be strictly below the critical density $\rho_{c}$.

Remark 2. For $u(t) \in\left[0, \rho_{c}\right)$, the boundary condition $\rho(t, 0)=u(t)$ is equivalent to $f(\rho(t, 0))=f(u(t))$. Thus, the boundary condition can be implemented by controlling the influx of vehicles per second as in ramp metering (see, e.g., [14-16]).

For a given upper bound $\rho_{c}-\varepsilon$ for the values of the boundary density and given values of the Lipschitz constants $L_{\rho_{0}}$ and $L_{u}$, Theorem 3 guarantees the existence of the 
solution without shocks only on a possibly short time interval $[0, T]$.

In the definition of (S) and thus also in Theorem 3 the boundary conditions at $x=l$ are not considered. If congested flow occurs at $x=l$, a shock wave might travel backwards into the region $[0, T] \times[0, l]$, thus interfering with the regular solution that is constructed in Theorem 3 . To avoid this effect, throughout the paper we assume free outflow at $x=l$ on the whole time interval $[0, T]$; that is, we assume that $x=l$ is an absorbing boundary, where we do not impose boundary conditions.

Theorem 3 (quasilinear initial boundary value problem). Let $T>0$ and $\varepsilon \in\left(0, \rho_{c}\right)$ be given. Assume that the initial density $\rho_{0}$ is Lipschitz continuous on $[0, l]$ with the Lipschitz constant $L_{\rho_{0}}>0$ and that for all $x \in[0, l]$ one has $0 \leq \rho_{0}(x) \leq \rho_{c}$.

Assume that the boundary density $u$ is Lipschitz continuous on $[0, T]$ with the Lipschitz constant $L_{u}>0$ and that for all $t \in[0, T]$ one has $0 \leq u(t) \leq \rho_{c}-\varepsilon$.

Assume that the $C^{0}$-compatibility conditions between $\rho_{0}$ and $u$ hold: that is,

$$
\rho_{0}(0)=u(0) \leq \rho_{c}-\varepsilon
$$

Assume that

$$
T<\min \left\{\frac{1}{L_{f^{\prime}} L_{\rho_{0}}}, \frac{\underline{\Lambda}(\varepsilon)}{L_{f^{\prime}} L_{u}}\right\} .
$$

Then system $(\mathbf{S})$ has a solution on $[0, T] \times[0, l]$ in the sense of characteristics without shocks and rarefaction fans. In particular, the solution is continuous.

Proof of Theorem 3. We consider a solution of (S) in the sense of characteristics. We define the characteristic curves that intersect the $x$-axis at the time $t=0$; we have for $x \in[0, l]$ and $s \geq 0$

$$
\xi(s, x, 0)=x+s f^{\prime}\left(\rho_{0}(x)\right) .
$$

For the characteristic curves that intersect the axis $x=0$ at a time $t \in[0, T]$ we have for $s \geq 0$

$$
\xi(s, 0, t)=(s-t) f^{\prime}(u(t)) .
$$

Then for each point $(s, z) \in[0, T] \times[0, l]$ there exists a characteristic curve such that $\xi(s, x, 0)=z$ or such that $\xi(s, 0, t)=z$.

In order to obtain a well-defined solution in the sense of characteristics it is sufficient to show that $T$ is chosen in such a way that it is impossible that the characteristic curves intersect for $s \leq T$.

Then at each point $(s, z) \in[0, T] \times[0, l]$ the solution is well defined since the value of $\rho$ is constant along the characteristic curves.

There are three possibilities of how two different characteristic curves can intersect at a point $(s, z) \in[0, T] \times[0, l]$ :

(1) Two characteristic curves of type (6) intersect: the equation $\xi\left(s, x_{1}, 0\right)=\xi\left(s, x_{2}, 0\right)$ with $x_{1}, x_{2} \in[0, l]$ and $x_{1} \neq x_{2}$ implies $f^{\prime}\left(\rho_{0}\left(x_{1}\right)\right) \neq f^{\prime}\left(\rho_{0}\left(x_{2}\right)\right)$ and

$$
s=-\frac{1}{\left(f^{\prime}\left(\rho_{0}\left(x_{2}\right)\right)-f^{\prime}\left(\rho_{0}\left(x_{1}\right)\right)\right) /\left(x_{2}-x_{1}\right)} \text {. }
$$

Due to our regularity assumptions on $f^{\prime}$ and $\rho_{0}$, the function $x \mapsto f^{\prime}\left(\rho_{0}(x)\right)$ is Lipschitz continuous with the Lipschitz constant $L_{f^{\prime}} L_{\rho_{0}}$. Hence, (8) implies the inequality $|s| \geq 1 / L_{f^{\prime}} L_{\rho_{0}}$. Hence, if $T<1 / L_{f^{\prime}} L_{\rho_{0}}$, an intersection of this type cannot occur for $s \in[0, T]$.

(2) Two characteristic curves of type (7) intersect: the equation $\xi\left(s, 0, t_{1}\right)=\xi\left(s, 0, t_{2}\right)$ with $t_{1}, t_{2} \in[0, T]$ and $t_{1} \neq t_{2}$ implies

$$
s-t_{2}=\frac{f^{\prime}\left(u\left(t_{1}\right)\right)}{\left(f^{\prime}\left(u\left(t_{2}\right)\right)-f^{\prime}\left(u\left(t_{1}\right)\right)\right) /\left(t_{2}-t_{1}\right)} .
$$

Due to our regularity assumptions on $f^{\prime}$ and $u$, the function $t \mapsto f^{\prime}(u(t))$ is Lipschitz continuous with the Lipschitz constant $L_{f^{\prime}} L_{u}$. Thus, using the definition of $\underline{\Lambda}(\varepsilon)$ in (2) we arrive at the inequality

$$
\left|s-t_{2}\right| \geq \frac{\underline{\Lambda}(\varepsilon)}{L_{f^{\prime}} L_{u}} .
$$

Thus, if $T<\underline{\Lambda}(\varepsilon) / L_{f^{\prime}} L_{u}$, an intersection of this type is impossible for $s \in[0, T]$.

(3) Characteristic curves of type (6) intersect characteristic curves of type (7): for $t \in[0, T]$ and $x \in[0, l]$ the equation $\xi(s, 0, t)=\xi(s, x, 0)$ implies

$$
s=\frac{1}{\left(f^{\prime}(u(t))-f^{\prime}\left(\rho_{0}(x)\right)\right) /\left(t f^{\prime}(u(t))+x\right)} .
$$

Due to our compatibility assumption (4) we have

$$
\begin{aligned}
& \left|f^{\prime}(u(t))-f^{\prime}\left(\rho_{0}(x)\right)\right| \\
& \quad=\left|f^{\prime}(u(t))-f^{\prime}(u(0))+f^{\prime}\left(\rho_{0}(0)\right)-f^{\prime}\left(\rho_{0}(x)\right)\right| \\
& \quad \leq L_{f^{\prime}} L_{u} t+L_{f^{\prime}} L_{\rho_{0}} x \\
& \quad \leq L_{f^{\prime}} L_{u} t \frac{f^{\prime}(u(t))}{\underline{\Lambda}(\varepsilon)}+L_{f^{\prime}} L_{\rho_{0}} x \\
& \quad \leq \max \left\{L_{f^{\prime}} \frac{L_{u}}{\underline{\Lambda}(\varepsilon)}, L_{f^{\prime}} L_{\rho_{0}}\right\}\left(t f^{\prime}(u(t))+x\right) .
\end{aligned}
$$

Thus, we obtain the inequality

$$
|s| \geq \frac{1}{\max \left\{L_{f^{\prime}}\left(L_{u} / \underline{\Lambda}(\varepsilon)\right), L_{f^{\prime}} L_{\rho_{0}}\right\}} .
$$

If $T$ satisfies (5) an intersection of this kind cannot occur for $s \in[0, T]$.

Thus, we have proved Theorem 3.

Remark 4. If $\rho_{0}$ satisfies the assumptions of Theorem 3, the compatibility assumption (4) holds, $\rho_{0}(x) \leq \rho_{0}(0)$ for all $x \in(0, l)$, and the control function $u$ is continuous and increasing on $[0, T]$ with values in $\left(0, \rho_{c}\right)$, then the solution 
exists as a continuous solution in the sense of characteristics if $T<1 / L_{f^{\prime}} L_{\rho_{0}}$.

The reason is that in this case for intersections of the characteristic curves of the second type, if $t_{1}<t_{2}$ we have $s-t_{2}<0$; hence, $\xi\left(s, 0, t_{2}\right)<0$. Thus, there is no such intersection of the second type with $s \in[0, T]$ and $\xi\left(s, 0, t_{2}\right) \in$ $[0, l]$.

Moreover, since $u$ is increasing, $u(t)>\rho_{0}(x)$ for all $t \epsilon$ $(0, T], x \in(0, l]$. Hence, for intersections of the characteristic curves of the third type, we have $f^{\prime}(u(t))<f^{\prime}\left(\rho_{0}(x)\right)$; thus, in this case we have $s<0$, so intersections of this type do not occur with $s \in[0, T]$.

Example 5. Let $\rho_{\max }>0$ and $u_{\max }>0$ and for $\rho \in\left[0, \rho_{\max }\right]$ define

$$
f(\rho)=u_{\max } \rho\left(1-\frac{\rho}{\rho_{\max }}\right)
$$

(see [12] and the introduction in [17]). Then $\rho_{c}=(1 / 2) \rho_{\max }$ and $L_{f^{\prime}}=2\left(u_{\max } / \rho_{\max }\right)$.

Let $l=2$ and for $x \in[0, l]$ define the initial density:

$$
\rho_{0}(x)=\frac{1}{16}[1+\cos (\pi x+\pi)]+\frac{4-\pi}{20} x .
$$

Figure 1 shows the graph of the initial density on the space interval $[0,2]$.

Choose $\rho_{\max } \geq \max _{x \in[0, l]} \rho_{0}(x)$. We have $L_{\rho_{0}}=\pi / 16+(4-$ $\pi) / 20, \rho_{0}(0)=0$, and $f^{\prime}\left(\rho_{0}(0)\right)=u_{\max }$. Hence,

$$
\frac{1}{L_{f^{\prime}} L_{\rho_{0}}}=\frac{\rho_{\max }}{u_{\max }(\pi / 8+(4-\pi) / 10)} .
$$

For $\beta \in\left[0, \rho_{c}\right)$ choose

$$
L_{u} \leq u_{\max }\left(1-2 \frac{\beta}{\rho_{\max }}\right)\left(\frac{\pi}{16}+\frac{4-\pi}{20}\right) .
$$

For $t \geq 0$ define the control:

$$
u(t)= \begin{cases}L_{u} t & \text { if } t \in\left[0, \frac{\beta}{L_{u}}\right], \\ \beta & \text { if } t>\frac{\beta}{L_{u}} .\end{cases}
$$

Then the compatibility condition (4) holds and $u$ is Lipschitz continuous with the Lipschitz constant $L_{u}$.

Choose $\varepsilon=\rho_{c}-\beta$. Then we have $\underline{\Lambda}(\varepsilon)=f^{\prime}(\beta)$ and hence due to (17)

$$
\frac{1}{L_{f^{\prime}} L_{\rho_{0}}} \leq \frac{\underline{\Lambda}(\varepsilon)}{L_{f^{\prime}} L_{u}} .
$$

Theorem 3 implies that, for all

$$
T<\frac{\rho_{\max }}{u_{\max }(\pi / 8+(4-\pi) / 10)}
$$

(5) holds. In particular, the solution in the sense of characteristics exists without shocks and rarefaction fans on the time

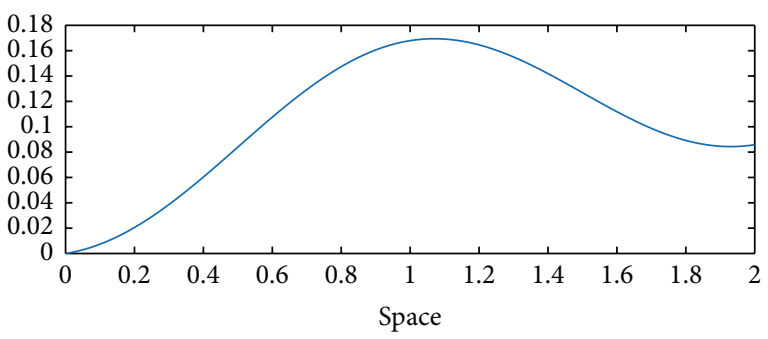

FIgure 1: The initial density $\rho_{0}$ for Example 5 on the space interval $[0,2]$.

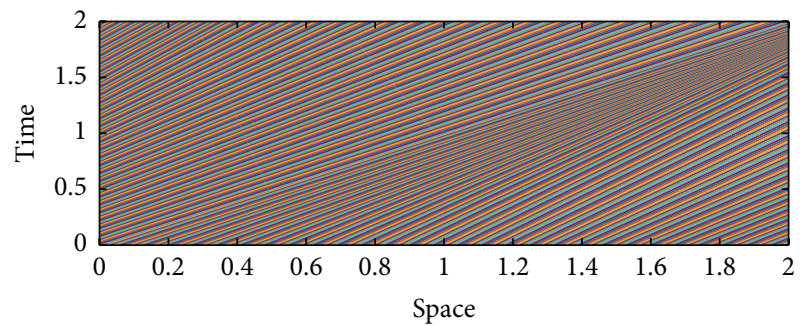

FIgURE 2: The characteristic curves for Example 5 generated with initial density $\rho_{0}, \rho_{\max }=1$, and $u_{\max }=1$ for $\beta=0.2$ on the time interval $[0,2]$. Since at time 2 the characteristic curves that contain the information of the initial density have already left the space interval $[0,2]$, this implies that with the constant control $u(t)=\beta$ for $t>2$ the solution continues to exist without shocks.

interval $\left[0,2\left(\rho_{\max } / u_{\max }\right)\right]$. If we choose $L_{u}$ as the right-hand side in (17), we have

$$
\frac{\beta}{L_{u}}<\frac{1}{L_{f^{\prime}} L_{\rho_{0}}}
$$

if and only if

$$
\beta<\frac{1}{4} \rho_{\max }
$$

Hence, if (22) holds the solution exists at least until $u(t)$ has attained the value $\beta$. Figure 2 shows the characteristic curves corresponding to the generated state $\rho(t, x)$ on $[0, T] \times[0, l]=$ $[0,2] \times[0,2]$ for $\rho_{\max }=1, u_{\max }=1, \beta=0.2$, and $L_{u}$ chosen as the right-hand side in (17). The horizontal axis is the space axis.

Example 6. Choose $f$ and $\rho_{0}$ as in Example 5. For $\omega>0$ define

$$
u(t)=\frac{L_{u}}{\omega}|\sin (\omega t)|
$$

(see Figure 3). Then the compatibility condition (4) holds and $u$ is Lipschitz continuous with the Lipschitz constant $L_{u}$. Choose $\varepsilon=\rho_{c}-L_{u} / \omega$. Then we have $\underline{\Lambda}(\varepsilon)=f^{\prime}\left(L_{u} / \omega\right)$. If

$$
L_{u} \leq \frac{u_{\max } L_{\rho_{0}}}{1+2\left(u_{\max } L_{\rho_{0}} / \omega \rho_{\max }\right)},
$$




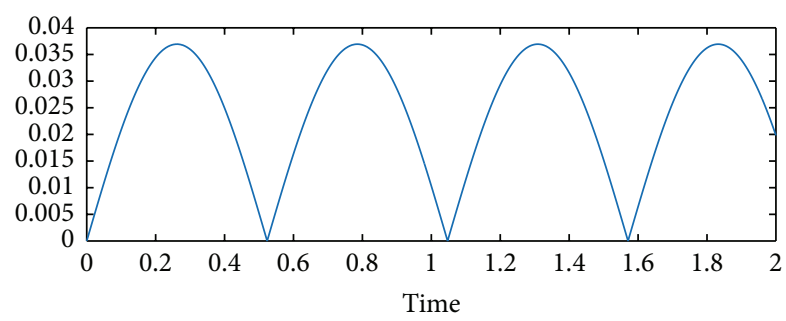

Figure 3: The function $u$ for Example 6 for $\omega=6$ and $L_{u}$ chosen as the right-hand side in (24) on the time interval $[0,2]$. The horizontal axis is the time axis.

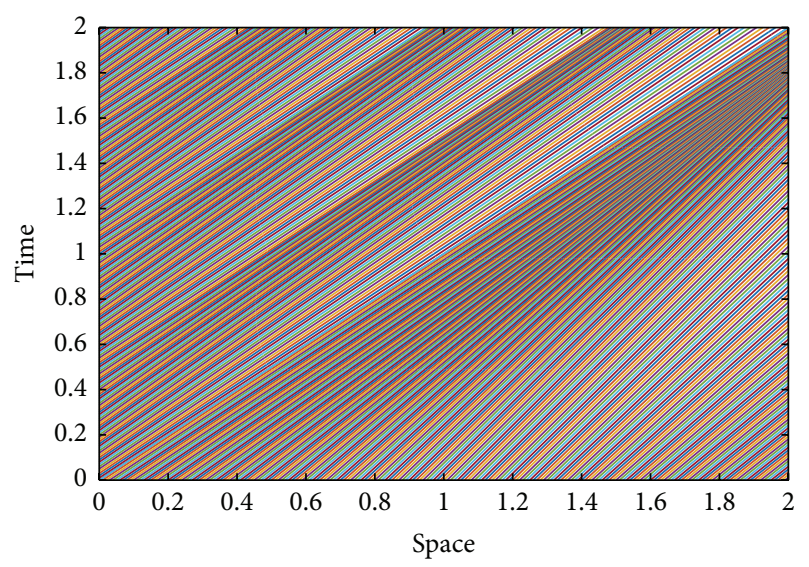

FIgURE 4: The characteristic curves for Example 6 generated with the initial density $\rho_{0}$ from Example 5, $\rho_{\max }=1, u_{\max }=1, \omega=6$, and $L_{u}$ chosen as the right-hand side in (24) on the time interval $[0,2]$.

inequality (19) holds. Hence, Theorem 3 implies that, for all $T>0$ that satisfy (20), a solution without shocks exists on $[0, T]$. In particular, such a solution exists on the time interval $\left[0,2\left(\rho_{\max } / u_{\max }\right)\right]$.

Figure 4 shows the characteristic curves corresponding to the state for $\rho_{\max }=1, u_{\max }=1, \omega=6$, and $L_{u}$ chosen as the right-hand side in (24) on the time interval $[0,2]$. Again the horizontal axis is the space axis $[0, l]$. The example illustrates that also oscillating inflow can generate a Lipschitz continuous solution if the Lipschitz constant of the boundary density is sufficiently small.

\section{Exact Controllability to Constant Densities}

Based on the solutions of (S) that we have obtained in Theorem 3, we can show that starting from a given initial density $\rho_{0}$ with values in $\left[0, \rho_{c}\right]$ we can control the traffic flow to any constant density $b<\rho_{c}$ in finite time. As before, this requires the assumption that at $x=l$ free traffic outflow occurs.

Theorem 7 (exact controllability to constant states). Assume that the initial density $\rho_{0}$ is Lipschitz continuous on $[0, l]$ with the Lipschitz constant $L_{\rho_{0}}$ and that for all $x \in[0, l]$ one has $0 \leq \rho_{0}(x) \leq \rho_{c}$ and $\rho_{0}(0)<\rho_{c}$. Moreover, assume that

$$
L_{\rho_{0}}<\frac{f^{\prime}\left(\rho_{0}(0)\right)}{l L_{f^{\prime}}} .
$$

Then for all $\beta \in\left(0, \rho_{c}\right)$ there exist a time $T>0$ and a piecewise linear Lipschitz continuous boundary control function $u(t)$ such that system (S) has a continuous solution on $[0, T] \times[0, l]$ without shocks in the sense of characteristics which satisfies for all $x \in[0, l]$ the end condition:

$$
\rho(T, x)=\beta .
$$

Thus, the traffic flow can be controlled arbitrarily close to a state of maximal throughput with a shock-free state.

To prove Theorem 7 , we show first that any initial state with a sufficiently small Lipschitz constant and $\rho_{0}(0)<\rho_{c}$ can be controlled to the constant state $\rho(x)=\rho_{0}(0)$ in finite time.

Theorem 8 (exact controllability to $\rho_{0}(0)$ ). Assume that the initial density $\rho_{0}$ is Lipschitz continuous on $[0, l]$ with the Lipschitz constant $L_{\rho_{0}}$ and that for all $x \in[0, l]$ one has $0 \leq \rho_{0}(x) \leq \rho_{c}$ and $\rho_{0}(0)<\rho_{c}$. Moreover, assume that

$$
L_{\rho_{0}}<\frac{f^{\prime}\left(\rho_{0}(0)\right)}{l L_{f^{\prime}}} .
$$

Let $T=l / f^{\prime}\left(\rho_{0}(0)\right)$. Then the constant control $u(t)=\rho_{0}(0)$ generates a continuous solution on $[0, T] \times[0, l]$ without shocks in the sense of characteristics for system (S) that satisfies for all $x \in[0, l]$ the end condition:

$$
\rho(T, x)=\rho_{0}(0) .
$$

Thus, the traffic flow can be controlled to a constant state without the generation of shocks.

Proof of Theorem 8. Let $\varepsilon=\rho_{c}-\rho_{0}(0)>0$. Define the control:

$$
u(t)=\rho_{0}(0) .
$$

Then $u$ is Lipschitz continuous for any Lipschitz constant $L_{u}>0$. Moreover, it satisfies the compatibility assumption (4) and $0 \leq u(t) \leq \rho_{c}-\varepsilon$. Hence, Theorem 3 implies that for all $T>0$ that satisfy (5), system (S) has a continuous solution on $[0, T] \times[0, l]$ without shocks in the sense of characteristics

The definition of $u$ implies that the slope of the characteristic curve that goes through $x=0$ at $t=0$ is $f^{\prime}\left(\rho_{0}(0)\right)$. Hence, the travel time until a characteristic curve that transports the values of the boundary density $u(t)$ reaches $x=l$ is

$$
\tau=\frac{l}{f^{\prime}\left(\rho_{0}(0)\right)} .
$$

Hence, if there exists a number $T>0$ that satisfies (5) and

$$
\tau \leq T,
$$


end condition (26) holds. Due to (30), this is equivalent to the inequality

$$
\frac{l}{f^{\prime}\left(\rho_{0}(0)\right)}<\min \left\{\frac{1}{L_{f^{\prime}} L_{\rho_{0}}}, \frac{f^{\prime}\left(\rho_{0}(0)\right)}{L_{f^{\prime}} L_{u}}\right\} .
$$

Due to assumption (27) we have

$$
\frac{l}{f^{\prime}\left(\rho_{0}(0)\right)}<\frac{1}{L_{f^{\prime}} L_{\rho_{0}}} .
$$

Moreover, we can choose $L_{u}>0$ such that

$$
L_{u}<\frac{\left(f^{\prime}\left(\rho_{0}(0)\right)\right)^{2}}{L_{f^{\prime}}} .
$$

Then (32) holds. Thus, we have proved Theorem 8.

Theorem 8 states that we can steer initial densities with sufficiently small Lipschitz constants to a constant state. Starting from this constant state, we can steer the system to any other constant state in $\left[0, \rho_{c}\right)$ that is sufficiently close to the initial state in finite time.

Theorem 9 (local exact controllability between constant states). Assume that the initial density is constant; that is, $\rho_{0}(x)=\alpha$ on $[0, l]$ with $\alpha \in\left[0, \rho_{c}\right)$. Let a number $\beta \in\left[0, \rho_{c}\right)$ be given. Assume that

$$
|\beta-\alpha|<\frac{\min \left\{f^{\prime}(\alpha), f^{\prime}(\beta)\right\}}{L_{f^{\prime}}} .
$$

Then there exist a time $T>0$ and piecewise linear Lipschitz continuous control $u(t)$ that generates a continuous solution on $[0, T] \times[0, l]$ without shocks in the sense of characteristics for system $(\mathbf{S})$ that satisfies for all $x \in[0, l]$ the end condition:

$$
\rho(T, x)=\beta .
$$

Thus, the traffic flow can be controlled between two freeflow constant states without the generation of shocks.

Proof of Theorem 9. Let $\varepsilon=\min \left\{\rho_{c}-\alpha, \rho_{c}-\beta\right\}>0$. For $L_{u}>0$ define the control:

$$
u(t)= \begin{cases}\alpha+\operatorname{sign}(\beta-\alpha) L_{u} t & \text { if } t \in\left[0, \frac{|\beta-\alpha|}{L_{u}}\right], \\ \beta & \text { if } t>\frac{|\beta-\alpha|}{L_{u}} .\end{cases}
$$

Then $u$ is Lipschitz continuous with the Lipschitz constant $L_{u}$. Moreover, it satisfies the compatibility assumption (4) and $0 \leq u(t) \leq \rho_{c}-\varepsilon$. Hence, Theorem 3 implies that (S) has a continuous solution on $[0, T] \times[0, l]$ without shocks in the sense of characteristics if $T$ satisfies (5). Moreover, the minimal slope of the characteristic curves that go through $x=$ 0 is $\underline{\Lambda}(\varepsilon)$; hence, the maximal travel time until a characteristic curve that transports the values of the boundary density $u(t)$ reaches $x=l$ is

$$
\tau_{\max }=\frac{l}{\underline{\Lambda}(\varepsilon)} .
$$

The definition of $u$ implies that, after the time $|\beta-\alpha| / L_{u}$, the travel time until a characteristic curve that transports the values of the boundary density $u(t)=\beta$ reaches $x=l$ is $l / f^{\prime}(\beta)$. Hence, if

$$
\frac{|\beta-\alpha|}{L_{u}}+\frac{l}{f^{\prime}(\beta)} \leq T,
$$

end condition (36) holds. Thus, it remains to find values of $\beta$ such that there exists a time $T$ that satisfies (39) and (5) at the same time. This is the case if

$$
\frac{|\beta-\alpha|}{L_{u}}+\frac{l}{f^{\prime}(\beta)}<\min \left\{\frac{1}{L_{f^{\prime}} L_{\rho_{0}}}, \frac{\underline{\Lambda}(\varepsilon)}{L_{f^{\prime}} L_{u}}\right\} .
$$

Since we can choose $L_{\rho_{0}}$ arbitrarily small, we only have to show that

$$
\frac{|\beta-\alpha|}{L_{u}}+\frac{l}{f^{\prime}(\beta)}<\frac{\underline{\Lambda}(\varepsilon)}{L_{f^{\prime}} L_{u}} .
$$

Assumption (35) implies that

$$
|\beta-\alpha|<\frac{\Lambda(\varepsilon)}{L_{f^{\prime}}}
$$

Thus, we can choose a number $L_{u}>0$ such that

$$
L_{u}<\frac{f^{\prime}(\beta)}{l}\left[\frac{\underline{\Lambda}(\varepsilon)}{L_{f^{\prime}}}-|\beta-\alpha|\right] .
$$

Then (41) holds. Thus, we have proved Theorem 9.

Example 10. In this example we study a decreasing control function for a constant initial state. Let $\rho_{0}(x)=\alpha \in\left(0, \rho_{c}\right)$ and $\beta \in[0, \alpha)$. Choose $L_{u}>0$.

For $t \geq 0$ define the control:

$$
u(t)= \begin{cases}\alpha-L_{u} t & \text { if } t \in\left[0, \frac{\alpha-\beta}{L_{u}}\right] \\ \beta & \text { if } t>\frac{\alpha-\beta}{L_{u}}\end{cases}
$$

Then compatibility condition (4) holds and $u$ is Lipschitz continuous with the Lipschitz constant $L_{u}$. Theorem 9 implies that if

$$
\alpha-\beta<\frac{f^{\prime}(\alpha)}{L_{f^{\prime}}},
$$

we can choose $L_{u}$ sufficiently small such that a solution in the sense of characteristics is generated. More precisely this is the case if $L_{u}$ satisfies (43): that is,

$$
L_{u}<\frac{f^{\prime}(\beta)}{l}\left[\frac{f^{\prime}(\alpha)}{L_{f^{\prime}}}-(\alpha-\beta)\right]
$$




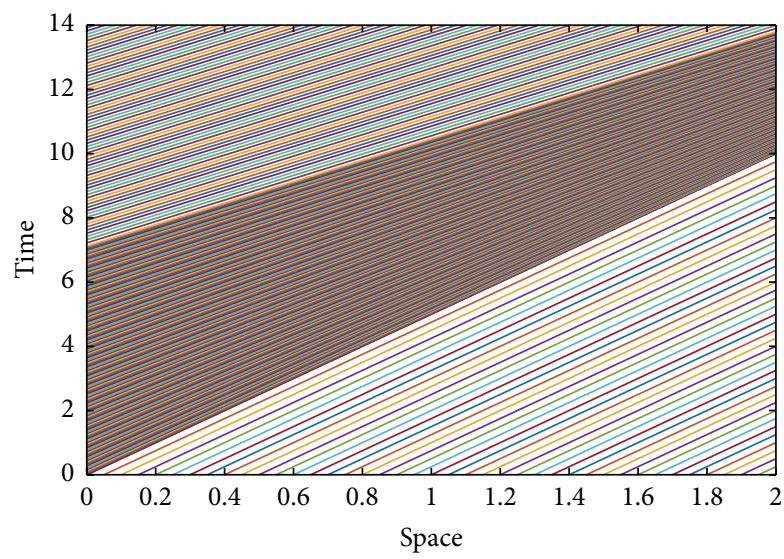

FIgURE 5: The characteristic curves for Example 10 generated with the initial density $\rho_{0}=0.4$ and $\beta=0.35, L_{u}=0.007, \rho_{\max }=1$, and $u_{\max }=1$.

and at the time $t^{*}=(\alpha-\beta) / L_{u}+l / f^{\prime}(\beta)$, the system has reached the state $\rho\left(x, t^{*}\right)=\beta$.

Figure 5 shows the characteristic curves corresponding to the state for $f$ as in Example 5: $\rho_{\max }=1, u_{\max }=1, \alpha=0.4$, $\beta=0.35$, and $L_{u}=0.007$ on the time interval $[0,14]$. Again the horizontal axis is the space axis $[0, l]$.

Example 11. In this example we study an increasing control function for a constant initial state. Let $\rho_{0}(x)=\alpha \in\left(0, \rho_{c}\right)$ and $\beta \in\left(\alpha, \rho_{c}\right)$. Choose $L_{u}>0$. For $t \geq 0$ define the control:

$$
u(t)= \begin{cases}\alpha+L_{u} t & \text { if } t \in\left[0, \frac{\beta-\alpha}{L_{u}}\right], \\ \beta & \text { if } t>\frac{\beta-\alpha}{L_{u}} .\end{cases}
$$

Then compatibility condition (4) holds and $u$ is Lipschitz continuous with the Lipschitz constant $L_{u}$. As pointed out in Remark 4, the control generates a solution without shocks and rarefaction fans for arbitrarily large $T$.

At the time $t^{*}=(\beta-\alpha) / L_{u}+l / f^{\prime}(\beta)$, the system has reached the state $\rho\left(x, t^{*}\right)=\beta$.

The generated solution is Lipschitz continuous. In fact for all $\left(t_{1}, x\right),\left(t_{2}, x\right) \in[0, T] \times[0, l]$, we have the inequality

$$
\left|\rho\left(t_{1}, x\right)-\rho\left(t_{2}, x\right)\right| \leq L_{u}\left|t_{1}-t_{2}\right| .
$$

For all $\left(t, \xi_{1}\right),\left(t, \xi_{2}\right) \in[0, T] \times[0, l]$, we have the inequality

$$
\left|\rho\left(t, \xi_{1}\right)-\rho\left(t, \xi_{2}\right)\right| \leq L_{u} \frac{1}{f^{\prime}(u(T))}\left|\xi_{1}-\xi_{2}\right| .
$$

This can be seen as follows. Let $\xi_{1}=\xi\left(t, 0, \tau_{1}\right)$ and $\xi_{2}=$ $\xi\left(t, 0, \tau_{2}\right)$ with $0 \leq \tau_{1}<\tau_{2} \leq t$. Then we have $\xi_{2}<\xi_{1}$ and

$$
\begin{aligned}
\tau_{2}- & \tau_{1} \\
& =\frac{\left(\tau_{1}-t\right)\left(f^{\prime}\left(u\left(\tau_{1}\right)\right)-f^{\prime}\left(u\left(\tau_{2}\right)\right)\right)+\left(\xi_{1}-\xi_{2}\right)}{f^{\prime}\left(u\left(\tau_{2}\right)\right)} .
\end{aligned}
$$

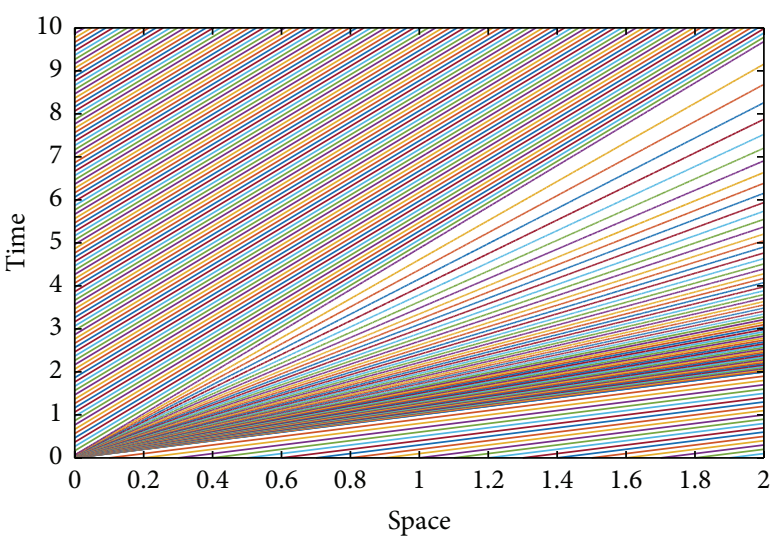

FIgURE 6: The characteristic curves for Example 11 generated with the initial density $\rho_{0}=0$ and $\beta=0.4, L_{u}=6, \rho_{\max }=1$, and $u_{\max }=1$.

Since $u$ is increasing, we have $f^{\prime}\left(u\left(\tau_{1}\right)\right)-f^{\prime}\left(u\left(\tau_{2}\right)\right) \geq 0$; thus, (50) implies

$$
0 \leq \tau_{2}-\tau_{1} \leq \frac{1}{f^{\prime}\left(u\left(\tau_{2}\right)\right)}\left(\xi_{1}-\xi_{2}\right) .
$$

Thus,

$$
\begin{aligned}
\left|\rho\left(t, \xi_{2}\right)-\rho\left(t, \xi_{1}\right)\right| & =\left|u\left(\tau_{2}\right)-u\left(\tau_{1}\right)\right| \leq L_{u}\left|\tau_{2}-\tau_{1}\right| \\
& \leq \frac{L_{u}}{f^{\prime}(u(T))}\left|\xi_{1}-\xi_{2}\right| .
\end{aligned}
$$

If one or both characteristic curves through $\left(t, \xi_{1}\right),\left(t, \xi_{2}\right)$ start at $\tau=0$ with $x>0$, where the initial density is prescribed, (49) also follows since $\rho_{0}$ is constant.

Due to (48) and (49), for all $\left(t_{1}, \xi_{1}\right),\left(t_{2}, \xi_{2}\right) \in[0, T] \times[0, l]$, we have the inequality

$$
\begin{aligned}
& \left|\rho\left(t_{1}, \xi_{1}\right)-\rho\left(t_{2}, \xi_{2}\right)\right| \\
& \quad \leq L_{u}\left[\left|t_{1}-t_{2}\right|+\frac{1}{f^{\prime}(u(T))}\left|\xi_{1}-\xi_{2}\right|\right] .
\end{aligned}
$$

Hence, the generated state is Lipschitz continuous and the size of the Lipschitz constant on $[0, T] \times[0, l]$ is proportional to $L_{u}$.

Figure 6 shows the characteristic curves corresponding to the state for $f$ as in Example 5: $\alpha=0, \rho_{\max }=1, u_{\max }=1$, $L_{u}=6$, and $\beta=0.4$ on the time interval $[0,10]$. Again the horizontal axis is the space axis $[0, l]$.

Using Theorems 8 and 9, we show that it is possible to steer initial densities with sufficiently small Lipschitz constants to any free-flow constant state.

Proof of Theorem 7. Starting with the constant control from Theorem 8, we can control the initial state in finite time to a constant state $a=\rho_{0}(0)$. On account of Theorem 9, it suffices that there exist a finite number of states $z_{0}, z_{1}, \ldots, z_{N} \in$ $[0, \max \{a, \beta\}]$ with $z_{0}=a$ and $z_{N}=\beta$ such that for all $i \in\{1, \ldots, N\}$ it is possible to steer the system from $z_{i-1}$ to $z_{i}$ in finite time with a piecewise linear Lipschitz continuous 
control. Inequality (35) in Theorem 9 implies that this is possible if for all $i \in\{1, \ldots, N\}$ we have the inequality

$$
\left|z_{i}-z_{i-1}\right| \leq \frac{\min \left\{f^{\prime}\left(z_{i}\right), f^{\prime}\left(z_{i-1}\right)\right\}}{L_{f^{\prime}}}
$$

Since for all $j \in\{0, \ldots, N\}$ we have

$$
f^{\prime}\left(z_{j}\right) \geq \min \left\{f^{\prime}(a), f^{\prime}(\beta)\right\},
$$

(54) holds if $z_{i}$ are chosen such that for all $i \in\{1, \ldots, N\}$ we have

$$
\left|z_{i}-z_{i-1}\right| \leq \frac{\min \left\{f^{\prime}(a), f^{\prime}(\beta)\right\}}{L_{f^{\prime}}}
$$

This is possible if $N$ is sufficiently large, for example, if the points $z_{i}$ are distributed equidistantly. Then we can reach the desired system state $b$ after $N$ intermediate control steps of the type described in Theorem 9. Thus, we have proved Theorem 7 .

\section{Lipschitz Constants for the State}

Up to now, we have shown that, for a given $\varepsilon>0$ that is sufficiently small, a continuous state is generated with suitable Lipschitz initial and boundary data. In this section we provide upper bounds for the Lipschitz constants of the generated states as functions of $\varepsilon$.

Theorem 12 (Lipschitz continuous states). Let $T>0$ and $\varepsilon \in$ $\left(0, \rho_{c}\right)$ be given. Assume that the conditions stated in Theorem 3 hold.

Define the numbers

$$
\begin{aligned}
& L_{a}=\frac{L_{u}}{\underline{\Lambda}(\varepsilon)-T L_{f^{\prime}} L_{u}}, \\
& L_{b}=\frac{L_{\rho_{0}}}{1-T L_{f^{\prime}} L_{\rho_{0}}} .
\end{aligned}
$$

Then the solution of $(\mathbf{S})$ on $[0, T] \times[0, l]$ is Lipschitz continuous in the sense that for all $\left(t_{1}, \xi_{1}\right),\left(t_{2}, \xi_{2}\right) \in[0, T] \times$ $[0, l]$ we have the inequality

$$
\begin{aligned}
& \left|\rho\left(t_{1}, \xi_{1}\right)-\rho\left(t_{2}, \xi_{2}\right)\right| \\
& \quad \leq\left[L_{a}+L_{b}\right]\left[\left|\xi_{1}-\xi_{2}\right|+\underline{\Lambda}(\varepsilon)\left|t_{1}-t_{2}\right|\right] .
\end{aligned}
$$

Proof of Theorem 12. Define the sets

$$
\begin{aligned}
& A_{a}=\{(s, x) \in[0, T] \times[0, l]: x \geq \xi(s, 0,0)\}, \\
& A_{b}=\{(s, x) \in[0, T] \times[0, l]: x \leq \xi(s, 0,0)\} .
\end{aligned}
$$

Then $A_{a} \cup A_{b}=[0, T] \times[0, l]$ and $S=A_{a} \cap A_{b}=\{(s, x) \in$ $[0, T] \times[0, l]: x=\xi(s, 0,0)\}$. We show that the solution $\rho$ of (S) is Lipschitz continuous on $A_{a}$ with the Lipschitz constant $L_{a}$ and $\rho$ is Lipschitz continuous on $A_{b}$ with the Lipschitz constant $L_{b}$ in the sense that for $j \in\{a, b\}$ and for all $\left(t_{1}, \xi_{1}\right)$, $\left(t_{2}, \xi_{2}\right) \in A_{j}$ we have the inequality

$$
\begin{aligned}
& \left|\rho\left(t_{1}, \xi_{1}\right)-\rho\left(t_{2}, \xi_{2}\right)\right| \\
& \quad \leq L_{j}\left[\left|\xi_{1}-\xi_{2}\right|+\underline{\Lambda}(\varepsilon)\left|t_{1}-t_{2}\right|\right] .
\end{aligned}
$$

Let $\left(t_{1}, \xi_{1}\right),\left(t_{2}, \xi_{2}\right) \in A_{a}$ be given. Then there exist points $x_{1}, x_{2} \in[0, l]$ such that $\xi_{1}=\xi\left(t_{1}, x_{1}, 0\right)$ and $\xi_{2}=\xi\left(t_{2}, x_{2}, 0\right)$. We have

$$
\begin{aligned}
\xi_{1}-\xi_{2}= & x_{1}-x_{2}+t_{1}\left[f^{\prime}\left(\rho_{0}\left(x_{1}\right)\right)-f^{\prime}\left(\rho_{0}\left(x_{2}\right)\right)\right] \\
& +\left(t_{1}-t_{2}\right) f^{\prime}\left(\rho_{0}\left(x_{2}\right)\right) .
\end{aligned}
$$

Hence, we have

$$
\begin{aligned}
\left|\xi_{1}-\xi_{2}\right| \geq & \left|x_{1}-x_{2}\right|-T L_{f^{\prime}} L_{\rho_{0}}\left|x_{1}-x_{2}\right| \\
& -\underline{\Lambda}(\varepsilon)\left|t_{1}-t_{2}\right| .
\end{aligned}
$$

Thus, we have

$$
\left|x_{1}-x_{2}\right| \leq \frac{1}{1-T L_{f^{\prime}} L_{\rho_{0}}}\left[\left|\xi_{1}-\xi_{2}\right|+\underline{\Lambda}(\varepsilon)\left|t_{1}-t_{2}\right|\right] .
$$

This yields

$$
\begin{aligned}
& \left|\rho\left(t_{1}, \xi_{1}\right)-\rho\left(t_{2}, \xi_{2}\right)\right|=\left|\rho_{0}\left(x_{1}\right)-\rho\left(x_{2}\right)\right| \\
& \quad \leq L_{\rho_{0}}\left|x_{1}-x_{2}\right| \\
& \quad \leq \frac{L_{\rho_{0}}}{1-T L_{f^{\prime}} L_{\rho_{0}}}\left[\left|\xi_{1}-\xi_{2}\right|+\underline{\Lambda}(\varepsilon)\left|t_{1}-t_{2}\right|\right] .
\end{aligned}
$$

Thus, we have shown (60) for $j=a$.

Let $\left(t_{1}, \xi_{1}\right),\left(t_{2}, \xi_{2}\right) \in A_{b}$ be given. Then there exist points $\tau_{1}, \tau_{2} \in[0, T]$ such that $\xi_{1}=\xi\left(t_{1}, 0, \tau_{1}\right)$ and $\xi_{2}=\xi\left(t_{2}, 0, \tau_{2}\right)$. We have

$$
\begin{aligned}
\xi_{1}-\xi_{2}= & \left(t_{1}-t_{2}\right) f^{\prime}\left(u\left(\tau_{1}\right)\right) \\
& +\left[t_{2}-\tau_{1}\right]\left[f^{\prime}\left(u\left(\tau_{1}\right)\right)-f^{\prime}\left(u\left(\tau_{2}\right)\right)\right] \\
& +\left(\tau_{2}-\tau_{1}\right) f^{\prime}\left(u\left(\tau_{2}\right)\right) .
\end{aligned}
$$

Hence, we have

$$
\begin{aligned}
\left|\xi_{1}-\xi_{2}\right| \geq & {\left[\underline{\Lambda}(\varepsilon)-\left|t_{2}-\tau_{1}\right| L_{f^{\prime}} L_{u}\right]\left|\tau_{1}-\tau_{2}\right| } \\
& -\underline{\Lambda}(\varepsilon)\left|t_{1}-t_{2}\right| .
\end{aligned}
$$

Thus, we obtain

$$
\begin{aligned}
& \left|\tau_{1}-\tau_{2}\right| \\
& \quad \leq \frac{1}{\underline{\Lambda}(\varepsilon)-T L_{f^{\prime}} L_{u}}\left[\left|\xi_{1}-\xi_{2}\right|+\underline{\Lambda}(\varepsilon)\left|t_{1}-t_{2}\right|\right] .
\end{aligned}
$$

This yields

$$
\begin{aligned}
& \left|\rho\left(t_{1}, \xi_{1}\right)-\rho\left(t_{2}, \xi_{2}\right)\right|=\left|u\left(\tau_{1}\right)-u\left(\tau_{2}\right)\right| \\
& \quad \leq L_{u}\left|\tau_{1}-\tau_{2}\right| \\
& \quad \leq \frac{L_{u}}{\underline{\Lambda}(\varepsilon)-T L_{f^{\prime}} L_{u}}\left[\left|\xi_{1}-\xi_{2}\right|+\underline{\Lambda}(\varepsilon)\left|t_{1}-t_{2}\right|\right] .
\end{aligned}
$$

Thus, we have shown (60) for $j=b$. 
For points $\left(t_{1}, \xi_{1}\right) \in A_{a},\left(t_{2}, \xi_{2}\right) \in A_{b}$ define the point $(\tilde{t}, \widetilde{\xi}) \in S$ as the point where the line that connects $\left(t_{1}, \xi_{1}\right)$ and $\left(t_{2}, \xi_{2}\right)$ intersects $S$. Then we have

$$
\begin{aligned}
& \left|\rho\left(t_{1}, \xi_{1}\right)-\rho(\tilde{t}, \tilde{\xi})\right| \leq L_{a}\left[\left|\xi_{1}-\tilde{\xi}\right|+\underline{\Lambda}(\varepsilon)\left|t_{1}-\tilde{t}\right|\right], \\
& \left|\rho\left(t_{2}, \xi_{2}\right)-\rho(\tilde{t}, \tilde{\xi})\right| \leq L_{b}\left[\left|\xi_{2}-\tilde{\xi}\right|+\underline{\Lambda}(\varepsilon)\left|t_{2}-\tilde{t}\right|\right] .
\end{aligned}
$$

This yields

$$
\begin{aligned}
& \left|\rho\left(t_{1}, \xi_{1}\right)-\rho\left(t_{2}, x_{2}\right)\right| \\
& \quad \leq\left|\rho\left(t_{1}, \xi_{1}\right)-\rho(\widetilde{t}, \widetilde{\xi})\right|+\left|\rho\left(t_{2}, \xi_{2}\right)-\rho(\widetilde{t}, \widetilde{\xi})\right| \\
& \quad \leq\left[L_{a}+L_{b}\right]\left[\left|\xi_{1}-x_{2}\right|+\underline{\Lambda}(\varepsilon)\left|t_{1}-t_{2}\right|\right] .
\end{aligned}
$$

Thus, we have shown (58) and Theorem 12 is proved.

Remark 13. Theorem 12 shows that the size of the Lipschitz constant $L_{a}+L_{b}$ of the state $\rho$ can be made arbitrarily small by the choice of $\varepsilon, T, L_{u}$, and $L_{\rho_{0}}$. small.

If $L_{\rho_{0}}$ is sufficiently small, $L_{b}>0$ can be made arbitrarily

Moreover, if $L_{u}$ is sufficiently small, $L_{a}>0$ can be made arbitrarily small.

Hence, in the situation of Theorem 8 , if $L_{\rho_{0}}$ is sufficiently small the Lipschitz constant of the generated state $\rho$ can be made arbitrarily small.

In the situation of Theorem $9, L_{b}>0$ can be chosen arbitrarily small. Due to (43) we can choose $L_{u}$ arbitrarily small. Hence, we can make $L_{a}$ arbitrarily small. Thus, we can control the system from a constant state $\alpha$ to a constant state $\beta$ in such a way that the Lipschitz constant $L_{a}+L_{b}>0$ of the generated state remains arbitrarily small.

Using the method described in Theorem 7, this implies that if $L_{\rho_{0}}$ is sufficiently small, it is possible to control the system from the initial state $\rho_{0}$ with $\rho_{0}(0)<\rho_{c}$ to a constant state $\beta \in\left(0, \rho_{c}\right)$ in such a way that the generated state is Lipschitz continuous and that the Lipschitz constant $L_{a}+L_{b}>$ 0 of the generated state remains arbitrarily small.

\section{The Lipschitz Controller}

For the convenience of the reader, we present the constructed Lipschitz continuous controller in explicit form. Let an initial density $\rho_{0}$ that satisfies the assumptions of Theorem 7 be given. Let a desired density $\beta \in\left[0, \rho_{c}\right)$ be given. Define $a=\rho_{0}(0)$.

We consider three cases.

(1) If $\beta=\rho_{0}(0)$, we can directly apply Theorem 8 where the constant control $u(t)=\rho_{0}(0)$ is used.

(2) The second case is $\beta \in\left(\rho_{0}(0), \rho_{c}\right)$. Define the density $z_{0}=a$ and the time $\tilde{t}_{0}=l / f^{\prime}(a)$ and let

$$
\begin{aligned}
& \widehat{t}_{1}=\widetilde{t}_{0}+\frac{\beta-a}{L_{u}}, \\
& \tilde{t}_{1}=\widehat{t}_{1}+\frac{l}{f^{\prime}(\beta)} .
\end{aligned}
$$

Similarly as in Example 11, for $L_{u}>0$ define the increasing control:

$$
u(t)= \begin{cases}a & \text { if } t \in\left[0, \tilde{t}_{0}\right], \\ a+L_{u}\left(t-\widetilde{t}_{0}\right) & \text { if } t \in\left(\widetilde{t}_{0}, \widehat{t}_{1}\right], \\ \beta & \text { if } t \in\left(\widehat{t}_{1}, \widetilde{t}_{1}\right] .\end{cases}
$$

Then $u$ is Lipschitz continuous with the Lipschitz constant $L_{u}$. Theorem 8 implies that, at the time $\widetilde{t}_{0}$, the system is in the constant state $a$. As pointed out in Example 11, at the time $\tilde{t}_{1}$ the system has reached the constant state $\beta$. Moreover, since the control is constant on the time interval $\left[0, \tilde{t}_{0}\right]$, Theorem 12 implies that the generated state is Lipschitz continuous on $\left[0, \widetilde{t}_{0}\right]$ with the Lipschitz constant:

$$
L^{*, 0}=L_{b}=\frac{L_{\rho_{0}}}{1-\widetilde{t}_{0} L_{f^{\prime}} L_{\rho_{0}}} .
$$

Note that due to (25), we have $L^{*, 0}>0$. In Example 11 we have stated that on the time interval $\left[\widetilde{t}_{0}, \widetilde{t}_{1}\right]$ the generated state is Lipschitz continuous in the sense of (53). In particular, the Lipschitz constant of the state can be made arbitrarily small by choosing $L_{u}$ arbitrarily small.

(3) Now we come to the third case where $\beta \in\left[0, \rho_{0}(0)\right)$. Here if the distance between the initial state and the target state is not sufficiently small, we have to steer the state through a certain number of intermediate states. In this way we construct a decreasing piecewise linear control that is constant on the intermediate states.

For this purpose, choose $z_{0}, z_{1}, z_{2}, \ldots, z_{N}$ such that $a=z_{0}>z_{1}>\cdots>z_{N}=\beta$ and a number $L_{u}>0$ such that for all $i \in\{1, \ldots, N\}$ we have

$$
L_{u}<\frac{f^{\prime}\left(z_{i}\right)}{l}\left[\frac{f^{\prime}\left(z_{i-1}\right)}{L_{f^{\prime}}}-\left(z_{i-1}-z_{i}\right)\right] .
$$

This can always be achieved with intermediate states on an equidistant grid, where for all $i \in\{1, \ldots, N\}$ we have $z_{i}=z_{0}+i((\beta-a) / N)$ if $N$ is sufficiently large. However, in order to reduce the number of intermediate states often it makes sense to increase the step size $\left(z_{i-1}-z_{i}\right)$ as $i$ increases, since $f^{\prime}\left(z_{i-1}\right)$ also increases with $i$.

Define the time $\widetilde{t}_{0}=l / f^{\prime}(a)$ and for all $i \in\{1, \ldots, N\}$ let

$$
\begin{aligned}
& \widehat{t}_{i}=\tilde{t}_{i-1}+\frac{z_{i-1}-z_{i}}{L_{u}}, \\
& \tilde{t}_{i}=\widehat{t}_{i}+\frac{l}{f^{\prime}\left(z_{i}\right)} .
\end{aligned}
$$


For $L_{u}>0$ define the control:

$$
\begin{aligned}
& u(t) \\
& = \begin{cases}a & \text { if } t \in\left[0, \widetilde{t}_{0}\right], \\
z_{i-1}-L_{u}\left(t-\widetilde{t}_{i-1}\right) & \text { if } t \in\left(\widetilde{t}_{i-1}, \widehat{t}_{i}\right], i \in\{1, \ldots, N\}, \\
z_{i} & \text { if } t \in\left(\widehat{t}_{i}, \widetilde{t}_{i}\right], i \in\{1, \ldots, N\} .\end{cases}
\end{aligned}
$$

Then $u$ is Lipschitz continuous with the Lipschitz constant $L_{u}$. Theorem 8 implies that, at the time $\widetilde{t}_{0}$, the system has reached the constant state $z_{0}$. For all $i \in$ $\{1,2, \ldots, N\}$, for the time interval $\left[\widetilde{t}_{i-1}, \widetilde{t}_{i}\right]$ due to $(74)$ the initial state $z_{i-1}$ and the terminal state $z_{i}$ satisfy (35). Moreover, condition (43) holds. Hence, the proof of Theorem 9 shows that, for all $i \in\{1,2, \ldots, N\}$, at the times $\widetilde{t}_{i}$ the system has reached the constant state $z_{i}$. Thus, at the terminal time

$$
T=\widetilde{t}_{N}=\frac{a-\beta}{L_{u}}+\sum_{j=0}^{N} \frac{l}{f^{\prime}\left(z_{i}\right)}
$$

end condition (26) holds for all $x \in[0, l]$. Moreover, since the control is constant on the time interval $\left[0, \widetilde{t}_{0}\right]$, Theorem 12 implies that the generated state is Lipschitz continuous on $\left[0, \widetilde{t}_{0}\right]$ with the Lipschitz constant:

$$
L^{*, 0}=L_{b}=\frac{L_{\rho_{0}}}{1-\widetilde{t}_{0} L_{f^{\prime}} L_{\rho_{0}}} .
$$

Note that due to (25), we have $L^{*, 0}>0$. For all $i \in\{1,2, \ldots, N\}$, define $\widetilde{T}_{i}=\widetilde{t}_{i}-\widetilde{t}_{i-1}$. For the time interval $\left[\widetilde{t}_{i-1}, \widetilde{t}_{i}\right]$ that has the length $\widetilde{T}_{i}$, the initial state is constant. Due to the construction in the proof of Theorem 9, $\widetilde{T}_{i}$ satisfies (5). Hence, Theorem 12 implies that the generated state is Lipschitz continuous on $\left[\widetilde{t}_{i-1}, \widetilde{t}_{i}\right]$ in the sense of (58) with the Lipschitz constant:

$$
L^{*, i}=\frac{L_{u}}{f^{\prime}\left(z_{i-1}\right)-\widetilde{T}_{i} L_{f^{\prime}} L_{u}}>0 .
$$

\section{Conclusions}

We have shown an exact controllability result for traffic flow described by the LWR model for free flow. We have shown that initial densities with sufficiently small Lipschitz constants can be steered to an arbitrary constant free-flow density in such a way that the Lipschitz constant of the generated state remains arbitrarily small. We have given the corresponding controllers explicitly. The density can be increased arbitrarily fast and still generate a Lipschitz continuous solution. However, in order to decrease the density and still generate a Lipschitz continuous solution some intermediate constant states are necessary.

The extension of this result to the case of road networks, for example, to study ramp metering, is a subject of future studies. Our results indicate that it makes sense to include upper bounds on the Lipschitz constant of the control functions as constraints in the corresponding optimal control problems. For the case of states where shocks are admitted, these problems have been studied, for example, in [14-16].

Recently, there has been a renewed interest in secondorder models for traffic flow; see $[17,18]$. In the two-phase models considered in [18], the uncongested phase is modelled by the LWR model, so this case is still covered by our results. It would be interesting to study whether the exact controllability results can also be adapted to the one-phase second-order models.

\section{Competing Interests}

The author declares that there are no competing interests.

\section{Acknowledgments}

This work was supported by DFG in the framework of the Collaborative Research Centre CRC/Transregio 154, Mathematical Modelling, Simulation and Optimization Using the Example of Gas Networks, Project C03, and by IPAM's long program "New Directions in Mathematical Approaches for Traffic Flow Management."

\section{References}

[1] M. J. Lighthill and G. B. Whitham, "On Kinematic Waves. II. A theory of traffic flow on long crowded roads," Proceedings of the Royal Society of London A, vol. 229, no. 1178, pp. 317-345, 1955.

[2] P. I. Richards, "Shock waves on the highway," Operations Research, vol. 4, pp. 42-51, 1956.

[3] R. M. Colombo and E. Rossi, "On the micro-macro limit in traffic flow," Rendiconti del Seminario Matematico della Università Di Padova, vol. 131, pp. 217-235, 2014.

[4] H. Greenberg, "An analysis of traffic flow," Operations Research, vol. 7, pp. 79-85, 1959.

[5] R. F. Cunningham and C. F. White, "Vehicular tunnel trafficflow control," IEEE Transactions on Vehicular Technology, vol. 19, no. 1, pp. 120-127, 1970.

[6] C. A. Lave, "Speeding, coordination, and the $55 \mathrm{MPH}$ limit," The American Economic Review, vol. 75, no. 5, pp. 1159-1164, 1985.

[7] M. Quddus, "Exploring the relationship between average speed, speed variation, and accident rates using spatial statistical models and GIS," Journal of Transportation Safety and Security, vol. 5, no. 1, pp. 27-45, 2013.

[8] T. Li, Controllability and Observability for Quasilinear Hyperbolic Systems, American Institute of Mathematical Sciences, Springfield, Ill, USA, 2010.

[9] M. Gugat, "Boundary controllability between sub- and supercritical flow," SIAM Journal on Control and Optimization, vol. 42, no. 3, pp. 1056-1070, 2003.

[10] J.-M. Coron, O. Glass, and Z. Wang, "Exact boundary controllability for 1-D quasilinear hyperbolic systems with a vanishing characteristic speed," SIAM Journal on Control and Optimization, vol. 48, no. 5, pp. 3105-3122, 2009/10.

[11] M. Gugat and G. Leugering, "Global boundary controllability of the de St. Venant equations between steady states," Annales 
de l'Institut Henri Poincare (C) Non Linear Analysis, vol. 20, no. 1, pp. 1-11, 2003.

[12] B. D. Greenshields, "A study of traffic capacity," Proceedings of the Highway Research Record, vol. 14, pp. 448-477, 1935.

[13] M. Gugat, Optimal Boundary Control and Boundary Stabilization of Hyperbolic Systems, SpringerBriefs in Electrical and Computer Engineering, Birkhäuser, Basel, Switzerland, 2015.

[14] H. Zhang, S. G. Ritchie, and W. W. Recker, "Some general results on the optimal ramp control problem," Transportation Research Part C, vol. 4, no. 2, pp. 51-69, 1996.

[15] D. Jacquet, C. C. De Wit, and D. Koenig, "Optimal ramp metering strategy with extended LWR model, analysis and computational methods," in Proceedings of the 16th Triennial World Congress of International Federation of Automatic Control (IFAC '05), pp. 99-104, Prague, Czech Republic, July 2005.

[16] J. Reilly, S. Samaranayake, M.-L. Delle Monache, W. Krichene, P. Goatin, and A. M. Bayen, "Adjoint-based optimization on a network of discretized scalar conservation laws with applications to coordinated ramp metering," Journal of Optimization Theory and Applications, vol. 167, no. 2, pp. 733-760, 2015.

[17] B. Seibold, M. R. Flynn, A. R. Kasimov, and R. R. Rosales, "Constructing set-valued fundamental diagrams from jamiton solutions in second order traffic models," Networks and Heterogeneous Media, vol. 8, no. 3, pp. 745-772, 2013.

[18] B. Piccoli, K. Han, T. L. Friesz, T. Yao, and J. Tang, "Second-order models and traffic data from mobile sensors," Transportation Research Part C, vol. 52, pp. 32-56, 2015. 


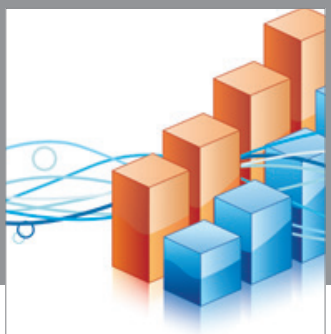

Advances in

Operations Research

vatem alat4

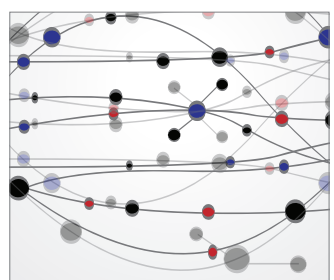

\section{The Scientific} World Journal
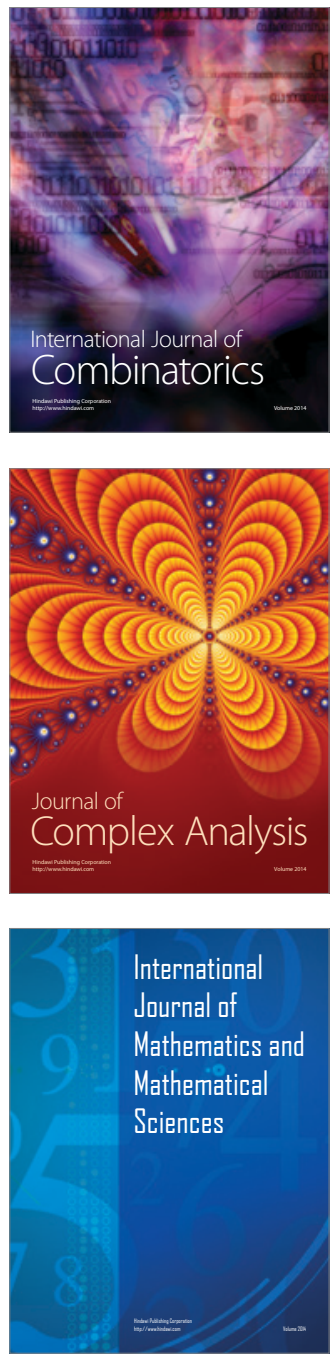
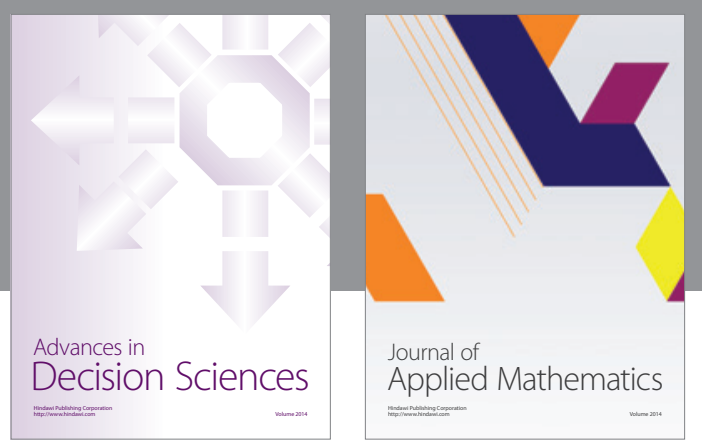

Algebra

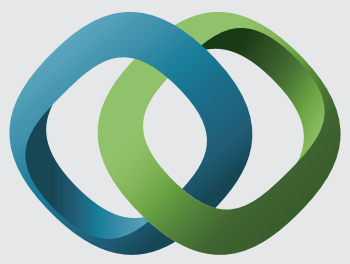

\section{Hindawi}

Submit your manuscripts at

http://www.hindawi.com
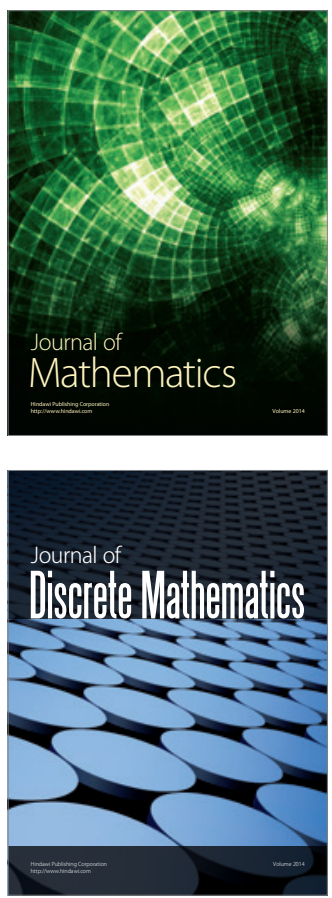

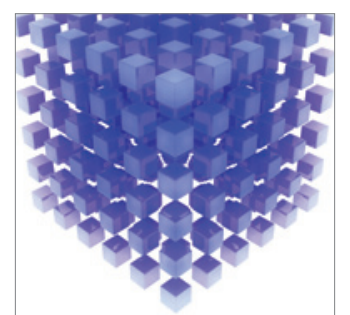

Mathematical Problems in Engineering
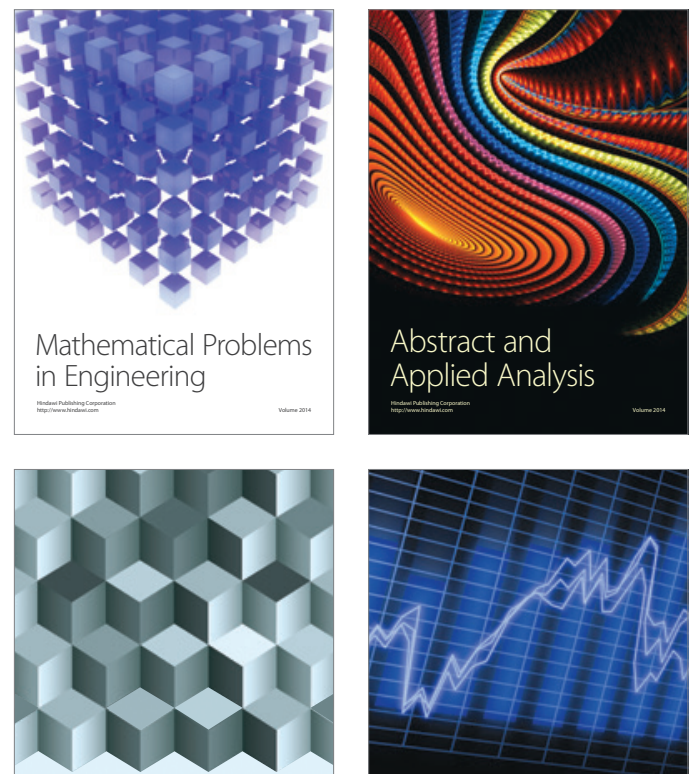

Journal of

Function Spaces

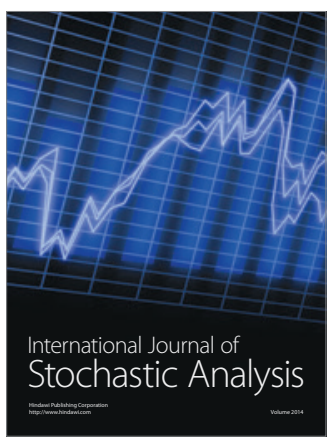

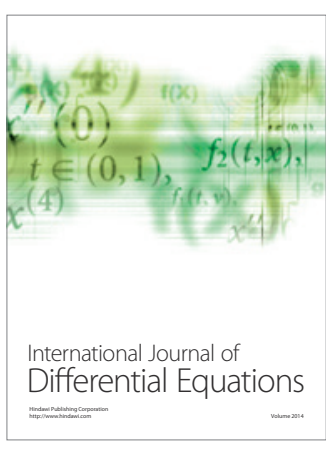
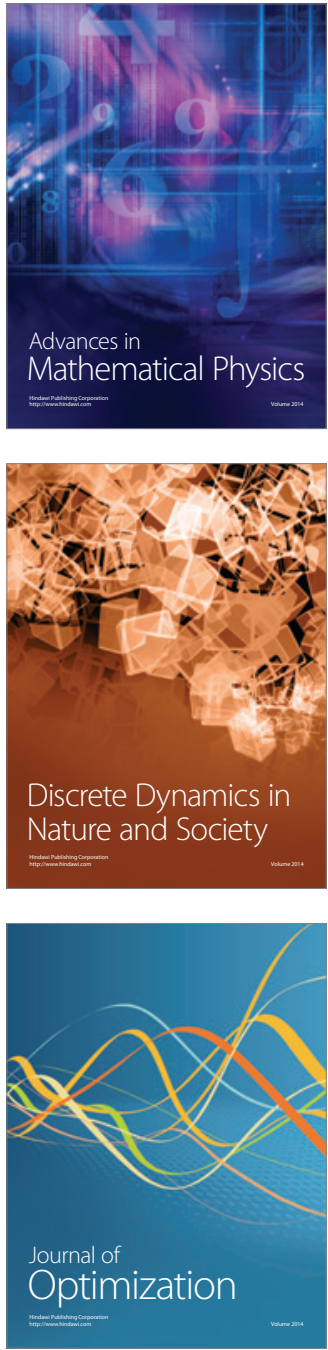